\title{
Improved error of electromagnetic shielding problems by a two-process coupling subproblem technique
}

\section{Dang Quoc Vuong*}

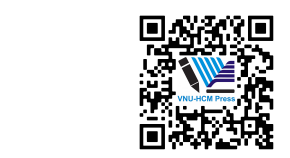

Use your smartphone to scan this QR code and download this article
Training Center of Electrical Engineering, School of Electrical Engineering, Hanoi, University of Science and Technology

\section{Correspondence}

Dang Quoc Vuong, Training Center of Electrical Engineering, School of Electrical Engineering, Hanoi, University of Science and Technology

Email: vuong.dangquoc@hust.edu.vn

History

- Received: 2020-04-03

- Accepted: 2020-05-11

- Published: 2020-05-18

DOI : 10.32508/stdj.v23i2.2054

\section{Check for updates}

\section{Copyright}

(c) VNU-HCM Press. This is an openaccess article distributed under the terms of the Creative Commons Attribution 4.0 International license.

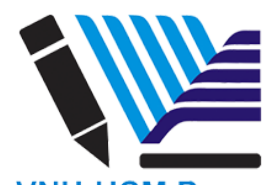

VNU-HCM Press

\begin{abstract}
Introduction: The direct application of the classical finite element method for dealing with magneto dynamic problems consisting of thin regions is extremely difficult or even not possible. Many authors have been recently developed a thin shell model to overcome this drawback. However, this development generally neglects inaccuracies around edges and corners of the thin shell, which leads to inaccuracies of the magnetic fields, eddy currents, and joule power losses, especially increasing with the thickness. Methods: In this article, we propose a two-process coupling subproblem technique for improving the errors that overcome thin shell assumptions. This technique is based on the subproblem method to couple SPs in two-processes. The first scenario is an initial problem solved with coils/stranded inductors together with thin region models. The obtained solutions are then considered as volume sources for the second scenario, including actual volume improvements that scope with the thin shell assumptions. The final solution is, to sum up, the subproblem solutions achieved from both scenarios. The extended method is approached for the $h$-conformal magnetic formulation. Results: The obtained results of the method are checked/compared to be close to the reference solutions computed from the classical finite element method and the measured results. This can be pointed out in a very good agreement. Conclusion: The extended method has also been successfully applied to the practical problem (TEAM workshop problem 21, model B).

Key words: Magnetic flux density, eddy current losses, Joule power losses, thin shells, finite element method, subproblem method (SPM)
\end{abstract}

\section{INTRODUCTION}

The direct application of the finite element method $(\mathrm{FEM})^{1}$ for dealing with magneto dynamic problems consisting of thin regions is extremely difficult or even not possible. Many authors ${ }^{2}$ have been recently developed a thin shell (TS) model in order to overcome this drawback. However, this development generally neglects inaccuracies around edges and corners of TS, which leads to inaccuracies of the local fields (magnetic fields, eddy currents, and Joule power losses...). The aim of this study is to propose a two-process coupling subproblem (SP) technique for improving the errors appearing from the TS models that were developed $\mathrm{in}^{2}$. The technique is herein based on the subproblem method (SPM) presented by many authors $^{3-7}$. The technique allows to couple SPs in twoprocesses. The first scenario is an initial problem solved with coils/stranded inductors and thin region models; the obtained solutions are then considered as volume sources (VSs) (express as of permeability and conductivity material in conducting regions) for the second scenario including actual volume improvements that scope with the TS assumptions ${ }^{2}$. The final solution is, to sum up, the SP solutions achieved from both the scenarios. The extended method is implemented for the magnetic field density formulation and applied to a practical problem (TEAM workshop Problem 21, model B) ${ }^{8}$.

\section{COUPLING SUBPROBLEM TECHNIQUE}

In the strategy SP, a canonical magneto dynamic problem $i$, to be solved at procedure $i$, is solved in a domain $\Omega_{i}$, with boundary $\partial \Omega_{i}=\Gamma_{i}=\Gamma_{h, i} \cup \Gamma_{b, i}$. The eddy current belongs to the conducting part $\Omega_{c, i}\left(\Omega_{c, i} \subset \Omega_{i}\right)$, whereas the stranded inductors are the non-conducting $\Omega_{C}^{C}$, with $\Omega_{c, i}=\Omega_{c, i} \cup \Omega_{c, i}^{C}$. The Maxwell's equations together with the following constitutive relations ${ }^{3-7}$.

$$
\begin{aligned}
& \text { curl } h_{\mathbf{i}}=j_{\mathbf{i}}, \operatorname{div} b_{\mathbf{i}}=0, \\
& \begin{array}{c}
\text { curle } e_{\mathbf{i}}=-\partial_{t} b_{\mathbf{i}} \\
b_{i}=\mu_{i} h_{\mathbf{i}}+b_{s, i}, \\
e_{i}=\sigma_{i}^{-1} j_{i}+e_{s, i} \\
n \times e_{i \mid \Gamma_{e}}=j_{f, i}
\end{array}
\end{aligned}
$$


where $\mathbf{b}_{i}$ is the magnetic flux density, $\mathbf{h}_{i}$ is the magnetic field, $\mathbf{e}_{i}$ is the electric field, $\mathbf{j}_{i}$ is the electric current density, $\mu_{\mathbf{i}}$ is the magnetic permeability, $\sigma_{i}$ is the electric conductivity and $\boldsymbol{n}$ is the unit normal exterior to $\Omega_{i}$. The surface field $j_{f, i}$ in $(2 \mathrm{c})$ is a surface source (SS) expressed as changes of interface conditions (ICs) and is generally defined as a zero for classical homogeneous boundary conditions (BCs). If nonzero, it can consider as SS that account for particular phenomena presenting at the idealized thin regions between the positive and negative sides of $\Gamma_{i}\left(\Gamma_{i}^{+}\right.$and $\left.\Gamma_{i}^{-}\right)$.

The source fields $\mathbf{b}_{s, i}$ and $\mathbf{e}_{s, i}$ in (2 a-b) are VSs. In the SPM, the changes of materials from the TS region ( $i=1, \mu_{1}$ and $\sigma_{1}$ ) to the volume improvement ( $i=$ $2, \mu_{2}$ and $\sigma_{2}$ ) can be defined via VSs ${ }^{4-7,9}$.

$$
\begin{aligned}
& b_{s, 2}=\left(\mu_{2}-\mu_{1}\right) h_{1}, \\
& e_{s, 2}=\left(\sigma_{2}^{-1}-\sigma_{1}^{-1}\right) j_{1}
\end{aligned}
$$

The total fields can be defined via a superposition method $^{1}$, i.e.

$$
\begin{aligned}
& b=b_{1}+b_{2}=\mu_{2}\left(h_{1}+h_{2}\right) \\
& e=e_{1}+e_{2}=\sigma_{2}^{-1}\left(j_{1}+j_{2}\right)
\end{aligned}
$$

\section{FINITE ELEMENT WEAK FORMULATION}

\section{Magnetic field intensity formulation}

By starting from the Ampere's law (1c), the weak conform magnetic field formulation of SP $i(i \equiv 1,2)$ can be written as ${ }^{3-7}$ :

$$
\begin{aligned}
& \partial_{t}\left(\mu_{i} h_{i}, h_{i}^{\prime}\right)_{\Omega_{i}}+\left(\sigma_{i}^{-1} \operatorname{curl} h_{i}, \operatorname{curl} h_{i}^{\prime}\right)_{\Omega_{\mathrm{c}, \mathrm{i}}} \\
& -\partial_{\mathrm{t}}\left(b_{s, i}, h_{i}^{\prime}\right)_{\Omega_{i}}+\left(e_{s, i}, \text { curl } h_{i}^{\prime}\right)_{\Omega_{i}} \\
& +<\left[n \times e_{i}\right]_{\gamma_{i}}, h_{i}^{\prime}>\Gamma_{i} \\
& +<n \times e_{i}, h_{i}^{\prime}>\Gamma_{i}-\gamma_{i}=0, \forall h_{i}^{\prime} \in H_{e, i}^{l}\left(\operatorname{curl}, \Omega_{i}\right) .
\end{aligned}
$$

The magnetic field $\boldsymbol{h}_{i}$ in (7) is decomposed into parts, $\boldsymbol{h}_{i}=\boldsymbol{h}_{s, i}+\boldsymbol{h}_{r, i}$, where $\boldsymbol{h}_{s, i}$ is the source magnetic field defined via an imposed electric current density in the stranded inductors $\Omega_{s, i}$, that is

$$
\begin{aligned}
& \left(\operatorname{curl} h_{s, i}, \operatorname{curl} h_{s, i}^{\prime}\right)_{\Omega_{s, i}}=\left(j_{s, i}, \operatorname{curl} h_{s, i}^{\prime}\right)_{\Omega_{s, i}}, \\
& \forall h_{s, i}^{\prime} \in H_{e, i}^{1}\left(\operatorname{curl}, \Omega_{s, i}\right)
\end{aligned}
$$

and $\boldsymbol{h}_{r, i}$ is the associated reaction magnetic field, which we have to define, i.e.

$$
\left\{\begin{array}{l}
\operatorname{curl} h_{s, i}=j_{s, i} \text { in } \Omega_{s, i} \\
\operatorname{curl} h_{r, i}=0 \text { in } \Omega_{c, i}^{C}-\Omega_{s, i}
\end{array}\right.
$$

In the non-conducting regions $\Omega_{c, i}^{C}$, the reaction field $\boldsymbol{h}_{r, i}$ is thus defined via a scalar potential ${ }^{7}$.
The function space $H_{e, i}^{1}(\ldots)$ in (7) and (8) is a curl - conform containing the basis functions for $\boldsymbol{h}_{i}$ and $\boldsymbol{h}_{s, i}$ as well as for the test function $h_{i}^{\prime}$ and $h_{s, i}^{\prime}$ (at the discrete level, this space is defined by finite edge elements); notations $(\cdot, \cdot)$ and $<\cdot, \cdot>$ are respectively a volume integral in and a surface integral of the product of their vector field arguments. The integral surface term

$<n \times e_{i}, h_{i}^{\prime}>\Gamma_{i}-\gamma_{i}$ on $\Gamma_{h}$ in (7) is defined as a homogeneous

Neumann BC, e.g., imposing a symmetry condition of "zero magnetic flux", i.e.

$$
n \times e_{i \mid \Gamma_{e}}=0 \Rightarrow n \bullet b_{i \mid \Gamma_{e, i}}=0 .
$$

The trace discontinuity $<\left[n \times e_{i}\right]_{\gamma_{i}}, h_{i}^{\prime}>_{\Gamma_{i}}$ appearing in (7) is considered as a TS model and given as ${ }^{1}$ :

$$
\begin{aligned}
& <\left[n \times e_{i}\right]_{\gamma_{i}}, h_{i}^{\prime}>_{\Gamma_{i}}=<\mu_{i} \beta_{i} \partial_{t}\left(2 h_{c, i}+h_{d, i}\right), h_{c, i}^{\prime}>_{\Gamma_{i}} \\
& +<\frac{1}{2}\left[\mu_{i} \beta_{i} \partial_{t}\left(2 h_{c, i}+h_{d, i}\right)+\frac{1}{\sigma_{i} \beta_{i}} h_{d, i}\right], h_{c, i}^{\prime}>_{\Gamma_{i}^{+}}
\end{aligned}
$$

where $\boldsymbol{h}_{c, i}$ and $\boldsymbol{h}_{d, i}$ are continuous and discontinuous components of $\boldsymbol{h}_{i}$, and $\beta_{i}$ is a factor defined as

$$
\begin{aligned}
& \beta_{i}=\gamma_{i}^{-1} \tanh \left(\frac{d_{i} \gamma_{i}}{2}\right), \\
& \gamma_{i}=\frac{1+j}{2}, \delta_{i}=\sqrt{\frac{2}{\omega \sigma_{i} \mu_{i}}}
\end{aligned}
$$

for $d_{i}$ and $\delta_{i}$ being the local thickness of the TS and skin depth, respectively.

\section{Projected solutions between thin shell and volume improvement}

The obtained solution $\boldsymbol{h}_{1}(\mathrm{i}=1)$ in sub-domain of the TS model $\Omega_{1}$ is now considered as a VS in a subdomain of the volume improvement (current problem) $\Omega_{2}$ (i=2). This means that at the discrete level, the source $\boldsymbol{h}_{1}$ solved in the mesh of the $\Omega_{1}$ has to be projected in mesh $\Omega_{2}$ via a projection method ${ }^{9}$. This can be done via its curl limited to $\Omega_{2}$, i.e.

$$
\begin{aligned}
& \left(\operatorname{curl} h_{1-2}, \operatorname{curl} \mathbf{h}_{2}^{\prime}\right)_{\Omega_{2}}=\left(\operatorname{curl} \mathbf{h}_{1}, \operatorname{curl} \mathbf{h}_{2}^{\prime}\right)_{\Omega_{2}}, \\
& \forall \mathbf{h}_{2}^{\prime} \in \mathrm{H}_{2}^{1}\left(\operatorname{Curl}, \Omega_{2}\right)
\end{aligned}
$$

Where $\mathrm{H}_{2}^{1}\left(\mathrm{Curl}, \Omega_{2}\right)$ is a gauged curl-conform function space for the projected source $\nabla_{1-2}$ and the test function $h_{2}^{\prime}$.

\section{Magnetic field intensity formulation with volume improvement}

The solution in (7) with the TS model (solved from the first scenario) is forced as a VS for solving the second problem (that contains an actual volume/volume improvement) through the volume integrals $\partial_{t}\left(b_{s, i}, h_{i}^{\prime}\right)_{\Omega_{\mathrm{i}}}$ and $\left(e_{s, i}, \operatorname{curl} a_{i}^{\prime}\right)_{\Omega_{i}}$, where $\boldsymbol{b}_{s, i} ; \boldsymbol{e}$ 
$s, i$ are given in (4a-b). For that, the weak formulation for a volume improvement (for example, $i=2$ ) is then written as

$$
\begin{aligned}
& \partial_{t}\left(\mu_{2} h_{2}, h_{2}^{\prime}\right)_{\Omega_{2}}+\left(\sigma_{2}^{-1} \operatorname{curl} h_{2}, \operatorname{curl} h_{2}\right)_{\Omega_{c, i}} \\
& \left.+\partial_{t}\left(\mu_{2}-\mu_{1}\right) h_{1}, h_{2}^{\prime}\right)_{\Omega_{2}} \\
& \left.+\left(\left(\sigma_{2}^{-1}-\sigma_{1}^{-1}\right) j_{1}, \operatorname{curl} h_{2}^{\prime}\right)_{\Omega_{2}}\right) \\
& +\left\langle n \times e_{2}, h_{2}^{\prime}\right\rangle_{\Gamma_{2}}=0, \forall h_{2}^{\prime} \in H_{e, 2}^{1}\left(\operatorname{curl}, \Omega_{2}\right) .
\end{aligned}
$$

At the discrete level, the source fields $\boldsymbol{h}_{1}$ and $\boldsymbol{j}_{1}$ defined in the mesh of the TS model $(i=1)$ via (7) are now projected in the mesh of the current SP/volume improvement SP $(i=2)$ via (13) shown in Section Projected solutions between thin shell and volume improvement.

\section{APPLICATION TEST}

The test problem is a TEAM workshop problem 21 $(\text { model B })^{8}$, with two excitation coils and a magnetic steel plate (Figure 1). The thickness of the plate is 10 $\mathrm{mm}$, and the electric conductivity is $\sigma=6.484 \mathrm{MS} / \mathrm{m}$, the relative magnetic permeability $\mu=200$, the frequency $\mathrm{f}=50 \mathrm{~Hz}$, and the exciting current of $25 \mathrm{~A}$. The test problem is solved in a 3-D case.

The distribution of the magnetic flux density $\boldsymbol{b}$ in a cut-plane due to the exciting/imposed current in the coils with a simplified mesh of the TS SP is shown in Figure 2.

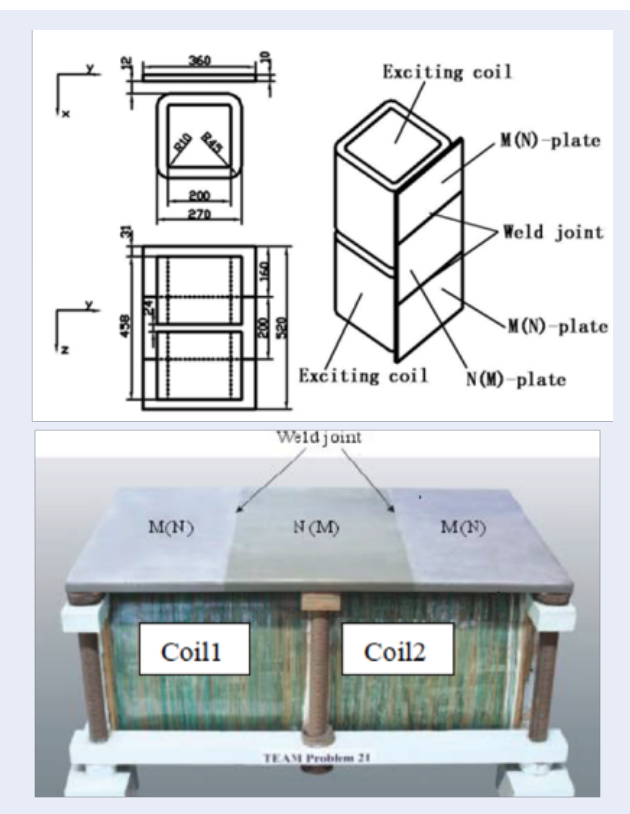

Fig. 1: The Geometry of the 2-D and 3-D [3]

Figure 1: The Geometry of the 2-D and 3-D ${ }^{8}$.

The inaccuracies on the eddy current density and Joule power loss density between the TS and volume improvement along the vertical edge (z-direction), with effects of $\mu, \sigma$ and $f(\mathrm{~d}=10 \mathrm{~mm})$, are improved by important volume improvements shown in Figure 3. The significant errors near the edges and corners reach $40 \%$ with $\delta$ (skin-depth) $=2.1 \mathrm{~mm}$ and thickness $d=10 \mathrm{~mm}$ in Figure 3 (top), and 50\% in Figure $3_{\text {(bottom) }}$ as well. The volume improvements are then checked to be close to the reference solutions (computed from FEM) for different parameters in both Figure 3. The relative improvement of the power loss density along the thin plate is presented in Figure 4. It can obtain up to $65 \%$ near the edges and corners of the TS.

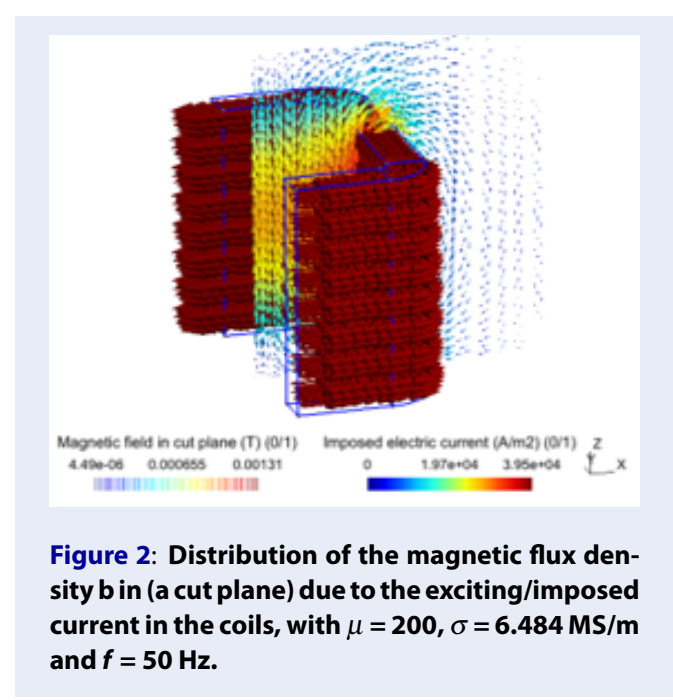

The results obtained on the magnetic flux density from the volume improvement are also compared with the measured results ${ }^{8}$ pointed out in Figure 5. The maximum and minimum errors between two method are proximately $10.9 \%$ and $1.5 \%$, respectively. This is said that there is a very suitable validation of the extended method.

\section{DISCUSSION AND CONCLUSION}

A two-process coupling subproblem technique with the magnetic field formulation has been successfully extended for improving errors on the local fields of magnetic flux density, eddy current density and Joule power loss density around the edges and corners of the TS approximations proposed in $^{2}$.

The obtained results of the method are checked to be close to the reference solution in computation of the classical FEM ${ }^{1}$ and are also compared to be similar the measured results from a TEAM workshop problem 21 (model, B) proposed by many authors ${ }^{8}$. This 


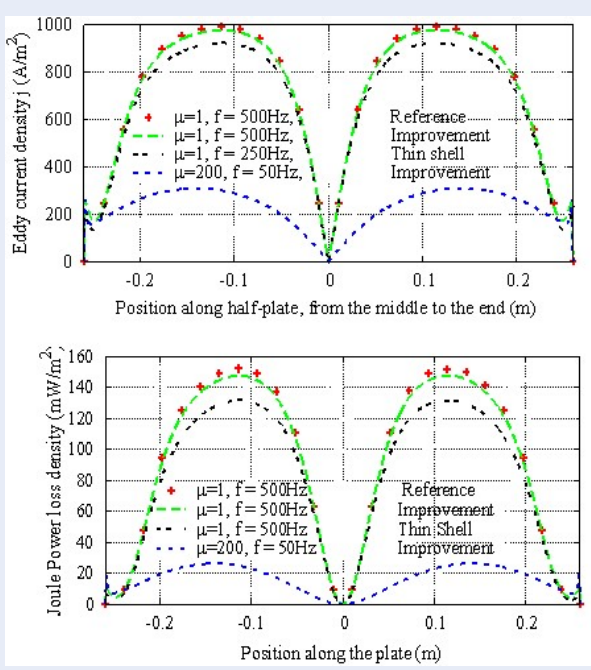

Figure 3: Eddy current density (top) and Joule power loss density (bottom) between the TS and volume solution along the vertical edge ( $z$ direction), with effects of $\mu, \sigma$ and $f(d=10 \mathrm{~mm})$.

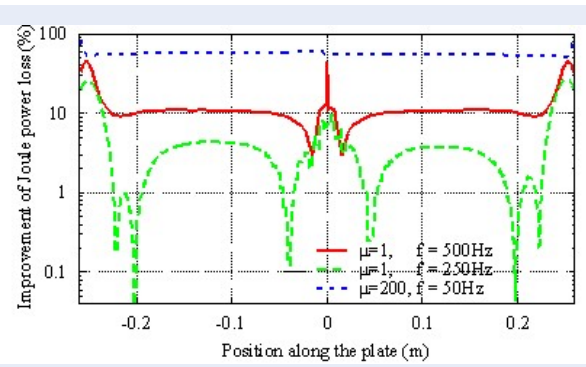

Figure 4: Relative improvement of the Joule power loss density along the plate, with the effects $\mu, \sigma$ and $\boldsymbol{f}(\mathrm{d}=\mathbf{1 0} \mathrm{mm})$.

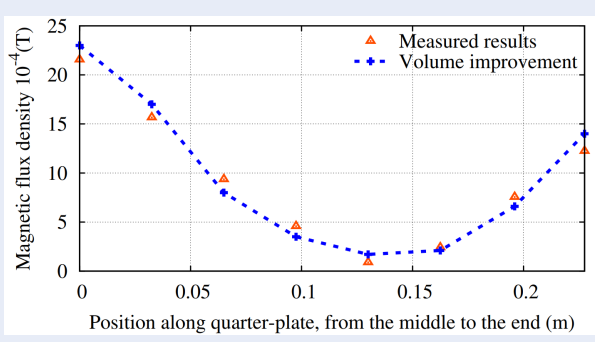

Figure 5: The comparison of the volume improvement (computed results) and measured results $^{3}$ along z-direction $\{x=0.576 \mathrm{~m}, y=0 \mathrm{~m}\}$.

is also demonstrated that there is a very good agreement between the studied technique and experiment methods.

The developed technique has been successfully carried out with the linear case in the frequency domain.
The extension of the method could be also implemented in the time domain and the nonlinear case (proposed in ${ }^{10}$ ) in next study.

All the steps of the technique have been validated and applied to international test problem (TEAM workshop problem 21 , model B) ${ }^{8}$. In particular, the achieved results is a good condition to analyze the influence of the fields to around electrical/electronic devices when taking a shielding plate into account.

\section{COMPLETING INTERESTS}

The author declares that there is no conflict of interest regarding the publication of this paper.

\section{AUTHOR'S CONTRIBUTIONS}

All the main contents, source-codes and the computed results of this article have developed by the author.

\section{REFERENCES}

1. Koruglu S, Sergeant P, Sabarieqo RV, Dang VQ, Wulf MD. Influence of contact resistance on shielding efficiency of shielding gutters for high-voltage cables. IET Electric Power Applications. 2011;5(9):715-720. Available from: https://doi.org/10. 1049/iet-epa.2011.0081.

2. Geuzaine C, Dular P, Legros W. Dual formulations for the Modeling thin Electromagnetic Shell using Edge Elements. IEEE Trans Magn. 2000;36(4):779-803. Available from: https://doi. org/10.1109/20.877566.

3. Dular P, Dang VQ, Sabariego RV, Krähenbühl L, Geuzaine C. Correction of thin shell finite element magnetic models via a subproblem method. IEEE Trans Magn. 2011;47(5):158 -1161. Available from: https://doi.org/10.1109/TMAG.2010.2076794.

4. Dang VQ, Dular P, Sabariego RV, Krähenbühl L, Geuzaine C. Subproblem approach for Thin Shell Dual Finite Element Formulations. IEEE Trans Magn. 2012;48(2):407-410. Available from: https://doi.org/10.1109/TMAG.2011.2176925.

5. Dang VQ, Dular P, Sabariego RV, Krähenbühl L, Geuzaine C. Subproblem Approach for Modelding Multiply Connected Thin Regions with an h-Conformal Magnetodynamic Finite Element Formulation. EPJ AP. 2013;63(1).

6. Dang VQ, Nguyen QD. Coupling of Local and Global Quantities by A Subproblem Finite Element Method - Application to Thin Region Models. Advances in Science, Technology and Engineering Systems Journal (ASTESJ). 2019;4(2):40-44. Available from: https://doi.org/10.25046/aj040206.

7. Dang VQ, Geuzaine C. Using edge elements for mdoeling of 3-D Magnetodynamic Problem via a Subproblem Method. Sci Tech Dev;23(1):439-445. Available from: https://doi.org/10. 32508/stdj.v23i1.1718.

8. Cheng Z, Takahash N, Forghani B. TEAM Problem 21 Family (V.2009). Approved by the International Compumag Society Board at Compumag-2009, Florianopolis, Brazil. 2009;.

9. Geuzaine C, Beys M, Henrotte F, Dular P, Legros W. A Galerkin projection method for mixed finite elements. IEEE Trans Magn. 1999;34(3):1438 -1441. Available from: https://doi.org/10. $1109 / 20.767236$

10. Sabariego RV, Geuzaine C, Dular P, Gyselnck J. Nonliner time domain finite element modeling of thin electromagnetic shells. IEEE Trans Magn. 2009;45(3):976 -979. Available from: https://doi.org/10.1109/TMAG.2009.2012491. 